\title{
Stiff self-interacting strings at high temperature QCD
}

\author{
A. S Bakry ${ }^{1, \star}$ X. Chen ${ }^{1}$, M. Deliyergiyev ${ }^{1}, A$. Galal $^{2}$, A. Khalaf ${ }^{2}$, and P. M Pengming ${ }^{1, \star \star}$ \\ ${ }^{1}$ Institute of Modern Physics, Chinese Academy of Sciences, Lanzhou 730000, China \\ ${ }^{2}$ Physics Dept., Faculty of Science, Al Azhar University, Cairo 11651, Egypt.
}

\begin{abstract}
.
We investigate the implications of Nambu-Goto (NG), Lüscher Weisz (LW) and Polyakov-Kleinert (PK) effective string actions for the Casimir energy and the width of the quantum delocalization of the string in 4-dim pure SU(3) Yang-Mills lattice gauge theory. At a temperature closer to the critical point $\mathrm{T} / \mathrm{Tc}=0.9$, we found that the next to leading-order (NLO) contributions from the expansion of the NG string in addition to the boundary terms in LW action to decrease the deviations from the lattice data in the intermediate distance scales for both the quark-antiquark $Q \bar{Q}$ potential and broadening of the color tube compared to the free string approximation. We conjecture possible stiffness of the QCD string through studying the effects of extrinsic curvature term in PK action and find a good fitting behavior for the lattice Monte-Carlo data at both long and intermediate quark separations regions.
\end{abstract}

\section{Introduction}

The formulation of an effective bosonic string description for gluonic degrees of freedom binding the hadron has been an attractive picture in consistency with with the main infrared (IR) features of quantum chromodynamics (QCD). The most simple choice relevant to the QCD string description is the NG string which is a bosonic string with an action taken proportional to the world-sheet area.

In the leading order approximation of the NG string action, the quantum fluctuations of the string bring forth a universal quantum correction to the linearly rising potential well known as the Lüscher term [1] in the meson and entail geometrical Lüscher-like terms [2] in the baryonic configuration. The width due to the quantum delocalizations of the string grows logarithmiclly $[3,4]$ as two color sources are pulled apart.

Precise lattice measurements of the $Q \bar{Q}$ potential in SU(3) gauge model are in consistency with the Lüscher subleading term for color source separation distance $R=0.5 \mathrm{fm}$ [1]. Many gauge models have unambiguously identified the Lüscher correction to the potential with unprecedented accuracy (for example [5-7]). The string model predicts, in addition, a logarithmic broadening [3] for the width profile of the string delocalization [8-11]

\footnotetext{
${ }^{\star}$ Speaker, e-mail: ahmed.bakry@mail.com

${ }^{\star}$ The authors acknowledge financial support by the Chinese Academy of Sciences President's International Fellowship Initiative grants No.2015PM062 and No.2016PM043, the Recruitment Program of Foreign Experts, NSFC grants (Nos. 11035006, 11175215, 11175220) and the Hundred Talent Program of the Chinese Academy of Sciences (Y101020BR0).
} 
Nevertheless, the simplified picture of the free bosonic string derived on the basis of the leading order formulation of NG action does not provide a good description of the numerical data in the intermediate distances and high temperatures. For instance, substantial deviations [12] from the free string behavior have been found for the lattice data corresponding to temperatures near the deconfinement point.

A comparison with the lattice Monte-Carlo data has shown the validity of the leading-order approximation at sources separation larger than $R=0.9 \mathrm{fm}[12,13]$ for both the quark-antiquark $(Q \bar{Q})$ potential and color-tube width profile. The region extends behind distances at which the leading-order string model predictions is valid [1] at zero temperature. In the baryon [14, 15], we found a similar behavior [16] for the long Y-string among quarks.

In this proceeding, we summarize the results obtained by studying the effects of the extrinsic curvature of the PK string in addition to the next to leading order expansion of the NG string action with boundary terms and the corresponding match to the lattice data. The numerical data of the potential and width profile of the energy density distribution is compare to the Casimir energy and the mean-square width of the string fluctuations of both PK and NG string action.

\section{String Phenomenology}

One particular form of string action that is Poincare and parity invariant is the Lüscher and Weisz string action. This form is introduced [1, 17] with additional built-in surface/boundary terms to account for the interaction of the open string with boundaries. The Lüscher and Weisz [11] (LW) effective action up to four-derivative term read

$$
S_{L W}[X]=\sigma A+\frac{\sigma}{2} \int d \zeta_{1} \int d \zeta_{2}\left[\left(\frac{\partial X}{\partial \zeta_{\alpha}} \cdot \frac{\partial X}{\partial \zeta_{\alpha}}\right)+\sigma \int d \zeta_{1} \int d \zeta_{2}\left[\kappa_{2}\left(\frac{\partial X}{\partial \zeta_{\alpha}} \cdot \frac{\partial X}{\partial \zeta_{\alpha}}\right)^{2}+\kappa_{3}\left(\frac{\partial X}{\partial \zeta_{\alpha}} \cdot \frac{\partial X}{\partial \zeta_{\beta}}\right)^{2}\right]+S_{b}\right.
$$

The vector $X^{\mu}\left(\zeta_{1}, \zeta_{2}\right)$ maps the region $C \subset \mathbb{R}^{2}$ into $\mathbb{R}^{D}$ and couplings $\kappa_{1}, \kappa_{2}$ are effective lowenergy parameters. Invariance under party transform would keep only even number derivative terms. The boundary term $S_{b}$ is relevant to the interaction of the effective string with the Polyakov loops at the fixed ends of the string and is given by

$$
S_{b}=\int d \zeta_{0}\left[b_{1} \partial_{2} X_{i} . \partial_{2} X^{i}+b_{2} \partial_{2} \partial_{1} X_{i} . \partial_{2} \partial_{1} X^{i}+\ldots . .\right]
$$

Consistency with the open-closed string duality [11] implies a vanishing value of the first boundary coupling $b_{1}=0$ in addition to a further constraint on the kinematically-dependent couplings in $D$ dimension given by $\kappa_{2}+\kappa_{3}=\frac{-1}{8 \sigma}$. This condition implies that all the terms with only first derivatives in the effective string action Eq. (1) coincide with the corresponding one in Nambu-Goto action to all orders in the derivative expansion.

The Nambu-Goto action is the most simple form of string actions proportional to area of the world-sheet, the action after gauge fixing reads

$$
S_{N G}[X]=\sigma \int_{0}^{L} d \zeta_{1} \int_{0}^{R} d \zeta_{2} \sqrt{\left(1+\left(\partial_{\zeta_{1}} X_{\perp}\right)^{2}+\left(\partial_{\zeta_{2}} X_{\perp}\right)^{2}\right)}
$$

A generalization of the Nambu-Goto string has been proposed by Polyakov [18] and Kleinert [19] to stabilize the NG action and was first investigated in the context of fluid membranes. This is a bosonic 
string with an extrinsic curvature as a next order operator after NG action. The action of the bosonic (Polyakov) string with the extrinsic curvature term reads

$$
S_{P K}=\frac{\sigma}{2} \int d^{2} \zeta \sqrt{g} g^{\alpha \beta} \partial_{\alpha} X . \partial_{\beta} X+\alpha_{r} \int d^{2} z \sqrt{g} \Delta X . \Delta X
$$

where $\Delta$ is Laplace operator. The extrinsic-curvature term filters out the sharply curved string configurations. The rigidity parameter provides the relative weight between the NG and this smoothing term in the effective string action [20]. Rigid string effects investigated first in Ref. [21] may manifest in the IR region of SU(N) non-abelian gauge theories [22] and could perhaps account for fine structure deviations from the simple NG string in quenched QCD.

The Casimir potential in $D$ dimensions is extracted from the string partition function which yields the first model-independent leading order contribution to the potential

$$
V_{\ell o}(R, T)=\sigma R+(D-2) T \ln \eta(\tau)+\mu(T),
$$

$\mu(T)$ is a renormalization parameter and $\eta$ is the Dedekind eta function with $\tau=\frac{i L_{T}}{R}$ is the modular parameter of the cylinder and $L_{T}=1 / T$ is the temporal extent governing the inverse temperature.

The mean-square width of the energy profile is defined as

$$
W^{2}(\xi ; \tau)=\frac{\int_{C}[D X] X^{2} \exp \left(-S_{N G}[X]\right)}{\int_{C}[D X] \exp \left(-S_{N G}[X]\right)}
$$

The mean-square width is calculated in Ref. [23] and would read as

$$
W_{\ell o}^{2}(\xi, \tau)=\frac{D-2}{2 \pi \sigma} \log \left(\frac{R}{R_{0}(\xi)}\right)+\frac{D-2}{2 \pi \sigma} \log \left|\frac{\theta_{2}(\pi \xi / R ; \tau)}{\theta_{1}^{\prime}(0 ; \tau)}\right|
$$

where $\theta$ are Jacobi elliptic functions with nome given as $q_{1}=e^{\frac{-\pi}{2} \tau}$, and $R_{0}(\xi)$ is the UV cutoff.

The next to leading corrections [24] to the Casimir energy from the explicit calculation of the two-loop approximation using $\zeta$ regularization scheme are given by

$$
V_{n \ell o}(R, T)=\sigma R+(D-2) T \ln \eta(\tau)-T \ln \left(1-\frac{(D-2) \pi^{2} T}{1152 \sigma_{o} R^{3}}\left[2 E_{4}(\tau)+(D-4) E_{2}^{2}(\tau)\right]\right)+\mu(T)
$$

The width of the NG string [25] at the next-to-leading order expansion of the action

$$
W_{N G}^{2}(\xi)=W_{\ell o}^{2}(\xi)+W_{n \ell o}^{2}(\xi)
$$

with the leading order term $W_{\ell o}^{2}$ is in accord to Eq. 7 and the next to leading term is given by

$$
\begin{array}{r}
W_{n \ell o}^{2}(\xi)=\frac{\pi}{12 \sigma R^{2}}\left[E_{2}(\tau)-4 E_{2}(2 \tau)\right]\left(W_{l o}^{2}(\xi)-\frac{D-2}{4 \pi \sigma}\right)+\frac{(D-2) \pi}{12 \sigma^{2} R^{2}}\left\{\tau\left(q_{2} \frac{d}{d q_{2}}-\frac{D-2}{12} E_{2}(\tau)\right)\right. \\
\left.\left[E_{2}(2 \tau)-E_{2}(\tau)\right]-\frac{D-2}{8 \pi} E_{2}(\tau)\right\}
\end{array}
$$

with $q_{2}=e^{-\pi \tau}$. The form of $W_{\ell o}^{2}$ in terms of Dedekind $\eta$ function given in Ref. [25] is equivalent to Eq. (7). 
The boundary term $S_{b}$ in Lüscher-Weisz action is of relevance to the interaction of the string with the boundaries. In Refs. [26, 27] the first nonvanishing Lorentz-Invariant boundary term contribution has been calculated. The modification to the potential for Dirichelet boundary condition is given by

$$
V_{b}=(d-2) b_{2} \frac{-\pi^{3} L_{T}}{60 R^{4}}
$$

$b_{2}$ is a fit parameter.

The fist-loop contribution of the extrinsic-curvature term at finite temperature [28]

$V_{S t i f f}^{\ell o}(R, T)=\sigma R+4 T \ln \eta(\tau)-2 T \sum_{n=0}^{\infty} \ln \left(1-e^{-2 R} \sqrt{(2 \pi n T)^{2}+\omega_{0}^{2}}\right)-\frac{\pi R T^{2}}{6}+\frac{T}{4} \ln \left(\frac{1}{(2 T R)}\right)+\mu(T),(12$

with $\omega_{0}=\frac{\sigma_{0}}{\alpha_{r i g}^{2}}$. The potential of the stiff string with the next to leading order NG contribution is

$$
V_{\text {Stiff }}^{n \ell o}(R, T)=V_{\text {Stiff }}^{\ell \circ}(R, T)-T \ln \left(1-\frac{(D-2) \pi^{2} T}{1152 \sigma_{o} R^{3}}\left[2 E_{4}(\tau)+(D-4) E_{2}^{2}(\tau)\right]\right),
$$

The mean-square width of the corresponding stiff string has been evaluated in detail in Ref. [29] and is given by

$$
W^{2}=W_{N G}^{2}+\frac{\pi(D-2) \alpha_{r}}{24 R^{2} \sigma^{2}} E_{2}\left(\frac{i L_{T}}{2 R}\right)
$$

\section{Numerical Results and Discussion}

\subsection{Monte-Carlo Simulations}

The Monte Carlo evaluation of the temperature-dependent quark-antiquark $(Q \bar{Q})$ potential at each $R$ is calculated through the Polyakov loop correlators

$$
\begin{aligned}
\mathcal{P}_{2 \mathrm{Q}} & =\int d[U] P(0) P^{\dagger}(R) \exp \left(-S_{w}\right), \\
& =\exp (-V(R, T) / T) .
\end{aligned}
$$

The two lattices employed in this investigation are of a typical spatial size of $3.6^{3} \mathrm{fm}^{3}$ with a lattice spacing $a=0.1 \mathrm{fm}$, temporal extents of $N_{t}=8$, and $N_{t}=10$ slices and coupling value of $\beta=$ 6.00. The two lattices correspond to temperatures $T / T_{c}=0.9$ just before the deconfinement point, and $T / T_{c}=0.8$ near the end of QCD plateau. The gauge configurations were generated using the standard Wilson gauge action employing a pseudo-heatbath algorithm updating the corresponding to three $S U(2)$ subgroup elements. The measurements are taken on 500 bins. Each bin consists of 4 measurements separated by 100 sweeps of updates.

A scalar field characterizing the action density in the Polyakov vacuum [30] is defined as

$$
C\left(\vec{\rho} ; \vec{r}_{1}, \vec{r}_{2}\right)=\frac{\left\langle\mathcal{P}_{2 Q}\left(\vec{r}_{1}, \vec{r}_{2}\right) S(\vec{\rho})\right\rangle}{\left\langle\mathcal{P}_{2 Q}\left(\vec{r}_{1}, \vec{r}_{2}\right)\right\rangle\langle S(\vec{\rho})\rangle}
$$

with the vector $\vec{\rho}$ refers to the spatial position of the energy probe with respect to some origin, and the bracket $<\ldots . . .>$ stands for averaging over gauge configurations and lattice symmetries. We choose a double Gaussian form [31] to estimate the mean-square width of the gluonic action-density (inlisted in detail in [31]). 


\subsection{Fit Analysis of the Potential of Static Meson}

To set comparison with the string model predictions, we fit the $Q \bar{Q}$ potential to that of the NG string Eqs.(5) and (8) for the exact expression of leading and next-to-leading order, separately. Similarly, we set the string tension $\sigma a^{2}$ and the renormalization constant $\mu(T)$ as free fitting parameters.

At temperature $T / T_{c}=0.8$, We found subtle changes in the fit behaviour of the NG string for both the free and self-interacting pictures to the lattice $Q \bar{Q}$ potential data with subtel differences in $\chi_{d o f}^{2}$ at the intermediate and the asymptotic large distances. We conclude that at zero and low temperatures up to $T / T_{c}=0.8$ the free string picture provides a good match with mesonic potential data in the IR region of QCD.

In spite of the improvement recieved by stretching out the fits to the NG string's self-interactions, the inclusion of the NLO terms does not provide an acceptable optimization for the potential data neither it does for the correct string tension dependency on the temperature near the deconfinement point $T / T_{c}=0.9$ (See Fig. 1). That is, the fit does not return a value for the free-parameter $\sigma_{0} a^{2}$ same as the one returned at $T / T_{c}=0.8$ or that measured at zero temperature [32].

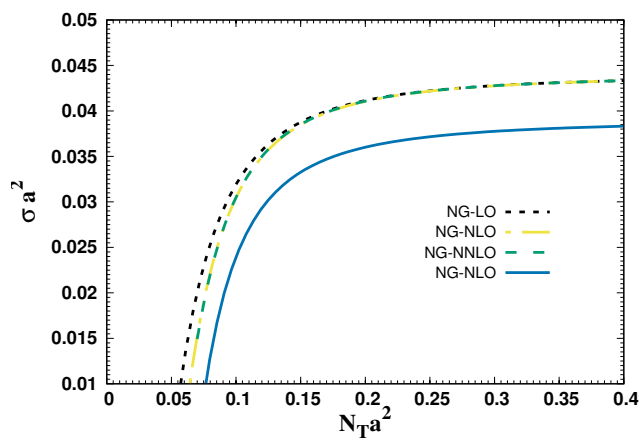

Figure 1. The temperature-dependence of the string tension for Nambu-Goto string action at LO, NLO and NNLO perturbative expansion. The dashed lines correspond to $\sigma_{0} a^{2}=0.044$ and solid line corresponds to $\sigma_{0} a^{2}=0.039$.

Effects such as the interaction of the string with the boundaries may be relevant to the discrepancy in the string description for intermediate distance and the correct string tension dependency on the temperature. The contribution to the Casimir energy due to the leading nonvanshing term $S_{b}$ in Lüscher-Weisz action Eq. (1) is given by Eq (11). The fits to Eq. (11) retun large values of $\chi^{2}$ when considering the fit interval $R \in[0.5,1.2] \mathrm{fm}$. Even though, nontrivial improvements in the values of $\chi^{2}$ are retrieved, as shown in Fig. 2, compared to that obtained [31] by merely considering the NG action Eqs. (5) and (8). Despite the reduction in the values of $\chi_{d o f}^{2}$, the consideration of the first boundary term Eq. (11) does not significantly alter the value of string tension at the minima of $\chi_{d o f}^{2}$. As seen in Fig. 2 the fit returns zero temperature string tension $\sigma_{0} a^{2}=0.0397$ with acceptable value of $\chi_{\text {dof }}^{2}$ on the fit interval $[0.7,1.2] \mathrm{fm}$.

Significant improvement in the fit behavior is observed when considering only the leading Eq. (12) together with stiffness effects. Even with the consideration of the stiffness correction together with the only leading-order NG potential Eq. (12) does provide a smaller $\chi_{d o f}^{2}$ compared to the fit to the LW static potential (NG potential at NLO Eq. (8) in addition to leading nonvanishing boundary term) Eq. (11) (See the discussion in [22]).

However, a precise match with the lattice data is obtained when taking into account the rigidity of the string together with the next to leading order term of NG string and the boundary terms of LW string. Figure. 3.2 depicts a very good agreement for the stiff string Casimir energy with the lattice $Q \bar{Q}$ data. That is, with the Use of all the approximation schemes Eqs. (5), (8), (11), (12) and (13).The 

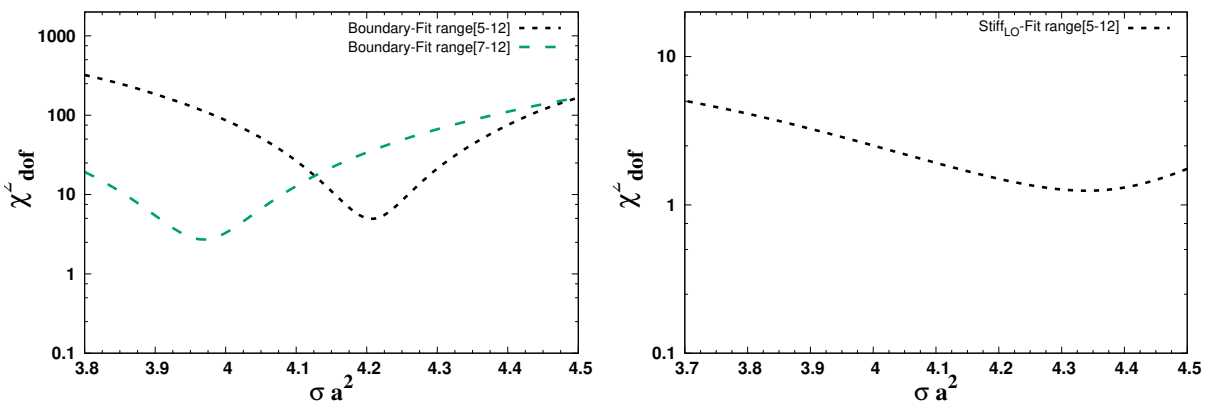

Figure 2. (a)The returned $\chi_{d o f}^{2}$ versus the string tension $\sigma a^{2}$ (scaled) from the fits of $Q \bar{Q}$ potential to NG string with boundary terms Eqs. (8) and (11) at $T / T_{c}=0.9$. (b)The returned $\chi^{2}$ from the fits to Eq. (13) and Eq. (11).
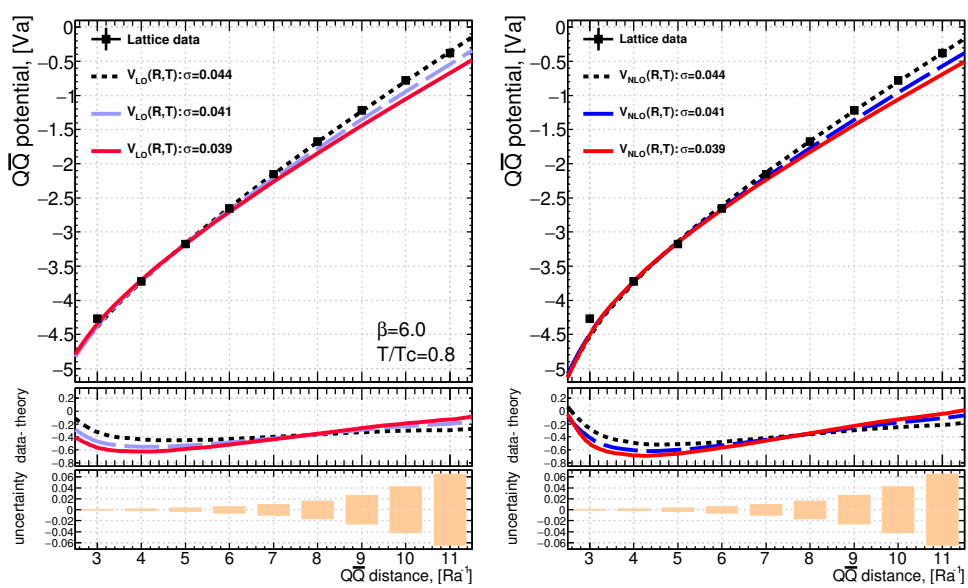

Figure 3. The $Q \bar{Q}$ potential at temperature $T / T_{c}=0.8$ and 0.9 , the lines correspond to the fits to the stiff string model Eq. (13) for the depicted values of $\sigma_{0}$.

plots of $\chi_{d o f}^{2}$ in Fig 2 show a minimal value returned at the same string tension value $\sigma_{0}$ measured at $T / T_{C}=0.8$.

\subsection{Fit Analysis of Energy-Density Width Profile}

In this section, the broadening of the width of the action density Eq. (16) at each selected transverse plane can be compared [31] to that of the corresponding width of the NG string Eqs.(7) and (10) for the leading and two-loop approximations, respectively. Similar to the numerical analysis of the $Q \bar{Q}$ data we show that the best fits to the energy profile are obtained when taking into account rigidity effects of the string together with the string self-interactions Eq. (14). 

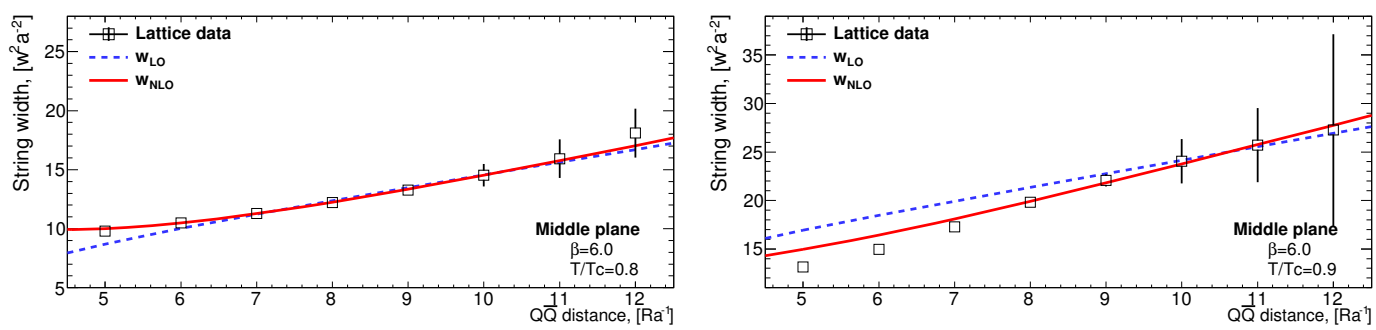

Figure 4. The mean-square width of the density distribution in the middle of the tube $z=R / 2$ at $T / T_{c}=0.9$ and $T / T_{c}=0.8$. The solid and dashed lines correspond to the free and self-interacting NG string Eq. (7) and Eq. (10), respectively.

Table 1. The returned $\chi^{2}$ from the fits of the action density width profile to Eq. (7), (10) and (14) using an optimal value of the rigidity $\alpha_{\text {rig }}$ returned from the fit of $Q \bar{Q}$ potential data to Eq. (13).

\begin{tabular}{llll}
\hline Fit range & $R \in[5,12]$ & $R \in[6,12]$ & $R \in[7,12]$ \\
\hline NG String (LO) & 82.98 & 53.30 & 15.71 \\
NG String (NLO) & 82.85 & 29.24 & 5.59 \\
Stiff String & 3.26 & 1.41 & 2.4 \\
\hline
\end{tabular}

In Table 1 the first two entries are the retuned value of $\chi^{2}$ considering fits of the string width profile in the middle plane to Eq. (7) and Eq. (10). These indicate improvements of the fits of the flux tube width in the middle plane compared the leading-order approximation of NG action.

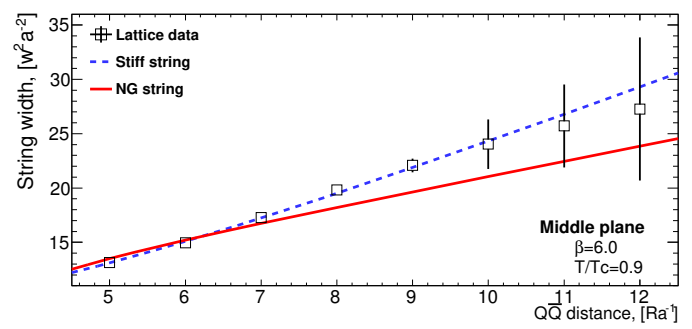

Figure 5. The mean-square width of the density distribution at the middle of the tube $z=R / 2$ and $T / T_{c}=0.9$. The solid and dashed lines correspond to the width of the Nambu-Goto and stiff string Eqs. (10) and (14), respectively.

Figure 4 compares the profile reproduced by the fits of the lattice data to the two approximation schemes of NG string at both temperatures $T / T_{c}=0.8$ and $T / T_{c}=0.9$. At the temperature $T / T_{c}=$ 0.8 , the fit [31] of the LO and NLO versions of NG string returns very close parameterization in both the asymptotic and intermediate distances regions regardless of the selected fit range. Indeed, higher order effects are almost absent at this temperature scale.

Even though, for short and long $Q \bar{Q}$ separation distances considered (Table (1)), the stiff string effects considered together with the next to leading expansion of the NG string provide the minimal value of $\chi^{2}$. The improvements in the fits with respect to pure NG string are obviously seen in Fig 5.

\section{Conclusion}

The theoretical predictions laid down by the free and self-interacting picture [31] are set into a comparison with the corresponding $S U(3)$ Yang-Mills lattice data in four space-time dimension. The region 
under scrutiny is the intermediate source separation $(R=0.5$ to $R=1.2 \mathrm{fm})$ for two temperatures scales; near the end of QCD plateau $T / T_{c}=0.8$, and just before the critical point $T / T_{c}=0.9$.

We conclude from the fit analysis of the $Q \bar{Q}$ potential and the energy width profile of the static meson the pure NG string does not provide precise match with lattice numerical data. However, we find a good agreement with the data, together with a subsequent retrieve of the correct string tension dependency on temperature, provided that the effects of the interaction with the boundaries and the rigidity of the string are taken into account in the portrayal of QCD string.

\section{References}

[1] M. Luscher, P. Weisz, JHEP 07, 049 (2002), hep-lat/0207003

[2] O. Jahn, P.D. Forcrand, Nucl. Phys. B - Proc. Suppl. 129-130, 700 (2004), lattice 2003

[3] M. Luscher, G. Munster, P. Weisz, Nucl. Phys. B180, 1 (1981)

[4] M. Pfeuffer, G.S. Bali, M. Panero, Phys. Rev. D 79, 025022 (2009)

[5] K.J. Juge, J. Kuti, C. Morningstar, Phys. Rev. Lett. 90, 161601 (2003), hep-lat/0207004

[6] N.H. Dass, P. Majumdar, Phys. Lett. B 658, 273 (2008)

[7] M. Caselle, M. Panero, D. Vadacchino, JHEP 02, 180 (2016), 1601.07455

[8] M. Caselle, F. Gliozzi, U. Magnea, S. Vinti, Nucl. Phys. B460, 397 (1996), hep-lat/9510019

[9] C. Bonati, Phys.Lett. B703, 376 (2011), 1106.5920

[10] M. Caselle, M. Hasenbusch, M. Panero, JHEP 03, 084 (2006), hep-lat/0601023

[11] M. Luscher, P. Weisz, JHEP 07, 014 (2004), hep-th/0406205

[12] A.S. Bakry et al., Phys. Rev. D 82, 094503 (2010,hep-lat/1004.0782)

[13] A.S. Bakry, D.B. Leinweber, A.G. Williams, Phys.Rev. D85, 034504 (2012), 1011.1380

[14] A.S. Bakry, X. Chen, P.M. Zhang, Phys. Rev. D91, 114506 (2015), 1412 . 3568

[15] A.S. Bakry, X. Chen, P.M. Zhang, AIP Conf. Proc. 1701, 030001 (2016)

[16] Bakry, Ahmed S., Chen, Xurong, Zhang, Peng-Ming, EPJ Web Conf. 126, 05001 (2016)

[17] M. Luscher, K. Symanzik, P. Weisz, Nucl. Phys. B173, 365 (1980)

[18] A. Polyakov, Nuclear Physics B 268, 406 (1986)

[19] H. Kleinert, Physics Letters B 174, 335 (1986)

[20] H. Kleinert, A. Chervyakov (1996), hep-th/9601030

[21] M. Caselle, M. Panero, R. Pellegrini, D. Vadacchino, JHEP 01, 105 (2015), 1406. 5127

[22] B.B. Brandt, JHEP 07, 008 (2017), 1705.03828

[23] A. Allais, M. Caselle, JHEP 01, 073 (2009), 0812 . 0284

[24] K. Dietz, T. Filk, Phys. Rev. D 27, 2944 (1983)

[25] F. Gliozzi, M. Pepe, U.J. Wiese, Phys.Rev.Lett. 104, 232001 (2010), 1002 . 4888

[26] O. Aharony, M. Field, JHEP 01, 065 (2011), 1008 . 2636

[27] M. Billo, M. Caselle, F. Gliozzi, M. Meineri, R. Pellegrini, JHEP 05, 130 (2012), 1202 . 1984

[28] V.V. Nesterenko, I.G. Pirozhenko, J. Math. Phys. 38, 6265 (1997), hep-th/9703097

[29] A.S. Bakry, X. Chen, M. Deliyergiyev, A. Galal, P.M. Zhang (2017), 1709.09446

[30] F. Bissey et al., Phys. Rev. D 76, 114512 (2007), hep-lat/0606016

[31] A. Bakry, X. Chen, M. Deliyergiyev, A. Galal, S. Xu, P.M. Zhang (2017), 1707.02962

[32] Y. Koma, M. Koma, Phys. Rev. D95, 094513 (2017), 1703.06247 